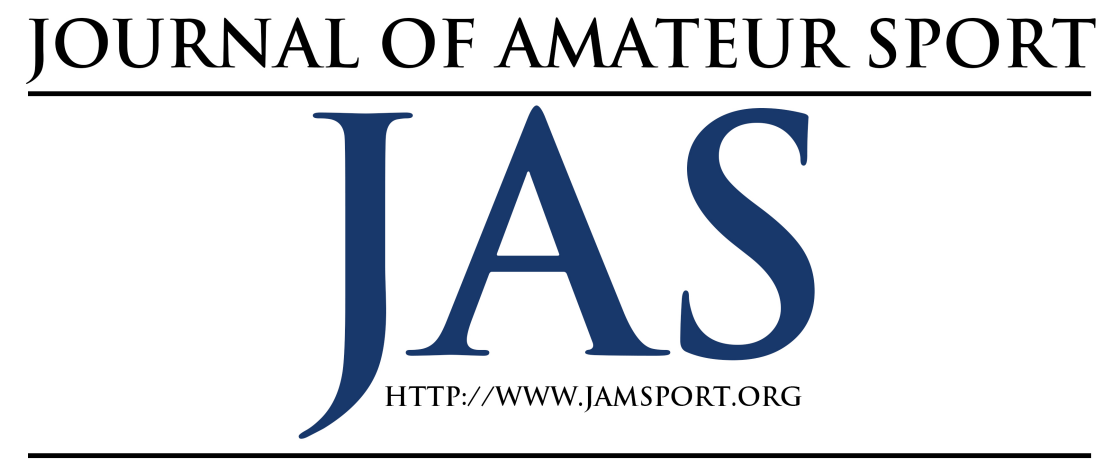

\title{
Examination of the 3x2 Achievement Goal Model in Collegiate Recreation: Comparison Across Sport Programs
}

\author{
Leeann M. Lower ${ }^{1}$ \\ Brian A. Turner ${ }^{2}$ \\ ${ }^{1}$ Ball State University \\ ${ }^{2}$ The Obio State University
}

Sport is a central achievement context through which participants' direct competence-related behavior to achieve desired outcomes. Achievement goal theory provides a framework for understanding self-directed behavior in achievement contexts. Theorists postulate that achievement goals reflect one's purpose and anticipated outcomes which guide behavior. To assess this psychological construct, several achievement goal measures have been developed, from a dichotomous model (Nicholls, 1989) to the most recent 3x2 model (Elliot, Murayama, \& Pekrun, 2011). In the present research, the $3 \times 2$ achievement goal model (i.e., task-approach, taskavoidance, self-approach, self-avoidance, other-approach, other-avoidance) was translated for the collegiate recreational sport domain and tested against alternative theoretical models. A questionnaire was developed and administered to recreational sport participants $(N=614)$ at a large, post-secondary institution in the United States. Using confirmatory factor analysis (CFA), the researchers found the $3 \times 2$ model to provide a better fit than the alternative theoretical models. These findings support the most recent theoretical advancement of the achievement goal construct. Through use of multivariate analysis of variance, significant differences across the six achievement goals were found between intramural and sport club participants. These results have implications for researchers and recreational sport practitioners seeking to measure and understand the motivational differences across sport participants.

central motivation construct
prevalent in sports psychology
literature is that of achievement goals, which are postulated to direct competence-related behavior (Conroy, Elliot, \& Hofer, 2003), such as sport 
involvement. Within the broader context of sport, there are several distinct sport models that are characteristic of diverse motivations. The achievement goals of collegiate sport athletes are likely to differ from that of collegiate recreational sport participants, which are subsequently likely to differ from that of youth sport participants. Collegiate recreation is a unique context in which to study achievement goals due to the diverse program offerings, including intramural sports, sport clubs, group fitness, aquatics, outdoor adventure, among other programs (Blumenthal, 2009). Through a greater understanding of the motivations of diverse recreation participants, practitioners will be more equipped to effectively design sport programming and influence participant behaviors.

Several theoretical models have been developed during the past three decades to conceptualize the achievement goal construct. More specifically, the literature highlights four primary models, including the dichotomous (Nicholls \& Dweck, 1979), trichotomous (Elliot \& Harackiewicz, 1996), 2x2 (Elliot \& McGregor, 2001), and 3x2 (Elliot et al., 2011) models. With the recent development of the $3 \times 2$ achievement goal framework, there is opportunity to study the achievement goals of recreational sport participants with greater precision, which will provide more information for practitioners to strategically design and deliver sport programs. The present research study was designed to examine the achievement goal construct within the collegiate recreational sport domain, with a dual-focus of (1) translating the $3 \times 2$ achievement goal model to the collegiate recreational sport context and testing the structure of the measurement model against alternative theoretical models, and (2) examining achievement goal differences across collegiate recreational sport groups.

\section{Achievement Goal Theory}

Achievement goal theory is a prominent motivation construct, which investigates the causes behind one's behavior (LeUnes, 2008). Achievement goals reflect one's purpose behind their behavior in achievement contexts as well as one's anticipated competence-related outcomes he/she hopes to attain or avoid from the behavior (Cury, Elliot, De Fonseca, \& Moller, 2006; Elliot, 2005; Roberts, Treasure, \& Conroy, 2007). The achievement goal construct, having emerged during the early 1980s, originally distinguished achievement goals by how competence is defined (Elliot, 2005). Nicholls (1984) postulated that two conceptions of ability (i.e., undifferentiated and differentiated) account for how competency is defined in achievement contexts, which then serves as the criteria used to assess successful achievement. An undifferentiated concept of ability does not distinguish between ability and effort, for which ability is evaluated through task mastery and personal improvement. In comparison, a differentiated concept of 
ability separates ability and effort, with the evaluation of ability based upon interpersonal comparison of effort and ability.

When considering the two conceptions of ability, an undifferentiated approach has been associated with mastery achievement goals, for which intrapersonal and absolute evaluation are used to determine successful achievement. Comparatively, the differentiated concept of ability aligns with performance achievement goals, which use interpersonal evaluation to assess success. Achievement goal theorists have also used the terms "task" and "ego" to reflect mastery and performance achievement goals respectively. As a whole, mastery achievement goals emphasize developing competence, while performance achievement goals focus on displaying competence (Cury et al., 2006; Elliot, 2005).

\section{Achievement Goal Models}

Several achievement goal models have emerged as the theory has evolved. The original dichotomous model differentiates achievement goals by definition of competency alone, without consideration of how competency is valenced (Elliot, 1999; Nicholls, 1984), resulting in omnibus achievement goals. As the theory has evolved, competence has been linked with approach and avoidance tendencies (Elliot \& Covington, 2001). Moreover, one either approaches the positive possibility of demonstrating competence, or avoids the negative possibility of demonstrating incompetence. The approach and avoidance tendencies towards competency are a function of valence. Models of the achievement goal construct have evolved to incorporate this valence component of competency to more effectively define the construct. Within achievement goal literature, four primary models have been developed and heavily used in empirical studies, including the dichotomous, trichotomous, $2 \times 2$, and $3 \times 2$ achievement goal models. Each model will be framed within the context of the $3 \times 2$ model tested in the current study, using 'task' and 'self to indicate mastery-based goals and 'other' to indicate performance-based goals.

Dichotomous model. Nicholls and Dweck (1979) are recognized for the development of the dichotomous achievement goal model (as cited in Elliot, 2005). The dichotomous model emphasizes mastery and performance achievement goals based upon how competency is defined, whereby all 'task' and 'self' items load onto a joint mastery latent factor and all 'other' items load onto a joint performance latent factor. Dweck and Leggett (1988) considered the achievement goal construct to be omnibus, in that both goals have approach and avoidance tendencies, while Nicholls (1984) characterized goal orientations as approach-oriented. Although still a prominent achievement goal framework, the dichotomous model has been criticized for failing to incorporate the approach-avoidance distinction commonly found in motivation theory (Elliot, 2005) 
Trichotomous model. The dichotomous model was expanded to incorporate the approach-avoidance distinction within the performance goal, creating a trichotomous achievement goal model, including mastery, performance approach, and performance avoidance goals (Elliot \& Harackiewicz, 1996). As there are variations of the trichotomous model, Conroy et al. (2003) labeled this specific trichotomous model tripartite model A, whereby all 'other' items load onto their hypothesized latent factors, and all 'task' and 'self' items onto a joint mastery latent factor. The mastery goal was considered approach-oriented based upon the supposition supported by achievement goal theorists that mastery goals focus on attaining competency and therefore facilitate positive outcomes (Elliot \& Harackiewicz, 1996; Nicholls, 1989). The trichotomous model has been criticized for its neglect of mastery-avoidance goals, which Yperen (2006) found to be a prominent achievement goal construct with almost one-third of respondents identifying mastery-avoidance as their dominant achievement goal.

$2 \times 2$ model. Elliot (2005) proposed the mastery achievement goal, without consideration of the approach-avoidance distinction, to be an inadequate measure of mastery goals as most measures only considered positive mastery possibilities. The tripartite model A was expanded to incorporate the approach-avoidance distinction within the mastery achievement goal, resulting in a $2 \times 2$ framework which includes mastery approach, mastery avoidance, performance approach, and performance avoidance goals (Elliot \& McGregor, 2001). The 2x2 model consists of all 'other' items loading onto their hypothesized latent factors, all taskapproach and self-approach items loading onto a joint mastery approach latent factor, and all task-avoidance and self-avoidance items loading onto a joint mastery avoidance latent factor. A criticism of the $2 \times 2$ model is that of the precision of the mastery-based goals, as they fail to differentiate an individual's focus on the task from their focus on personal improvement (Mascret, Elliot, \& Cury, 2014).

$3 \times 2$ model. Among the achievement goal models, competency has consistently been defined by mastery and performance achievement goals. To increase the precision of the model, Elliot et al. (2011) integrated absolute (task-based), intrapersonal (self-based), and interpersonal (other-based) standards of evaluation into the achievement goal model, in which mastery goals utilize task-based and selfbased evaluation and performance goals employ other-based evaluations. The achievement goal model was expanded to incorporate standards of evaluation based upon the possibility that one might pursue mastery goals focused on attaining either task-based or self-based competence independently. The 3x2 achievement goal model is an extension of the $2 \times 2$ 
framework, consisting of six distinct achievement goals, including mastery goals (task-approach, task-avoidance, selfapproach, self-avoidance) and performance goals (other-approach, other-avoidance).

Within the context of sport, there are conceptual, empirical, and practical reasons to adopt the $3 \times 2$ achievement goal framework over the alternative theoretical models (Mascret et al., 2014). The conceptual differentiation of task-based and self-based goals provide for greater precision in describing competence-related behavior. More specifically, individuals engaged in sport may focus on accomplishing the task at hand or improving their skills. Additionally, existing measures of mastery goals either emphasize task-based standards (Wang, Biddle, \& Elliot, 2007), self-based standards (Conroy et al., 2003), or a combination of both (Riou et al., 2012). This variation suggests that measures of mastery goals may actually be measuring different constructs, making subsequent interpretation difficult. From a practical standpoint, mastery goals are most often found associated with positive outcomes (Adie \& Jowett, 2010; Lower, Turner, \& Petersen, 2014; Nien \& Duda, 2008). Therefore, in order to effectively design sport programs to enhance mastery goals, a more precise understanding of the mastery achievement goal construct is necessary.

\section{Achievement Goals in Sport}

The achievement goal construct has been used in sport to better understand the psyche of an athlete and predict sportrelated behaviors. Achievement goal measures have been used to predict psychological and emotional functioning (Adie, Duda, \& Ntoumanis, 2010); cognitive anxiety (Kavussanu \& Morris, 2009); perceived performance (Stoeber, Uphill, \& Hotham, 2009); and feelings of success (Gilson, Chow, \& Ewing, 2008). When considering sport-related behaviors, the achievement goal framework has been found to be an important construct to explain disordered eating among female athletes (de Bruin, Bakker, \& Oudejans, 2009) and drop-out rates in youth sport (Cervelló, Excartí, \& Guzmán, 2007), among a variety of other behaviors. Overall, mastery and approach oriented goals are most frequently associated with positive outcomes, comparative to performance and avoidance goals (Kavussanu, White, Jowett, \& England, 2011; Lower et al., 2014; Spray, Wang, Biddle, \& Chatzisarantis, 2006). However, within certain sport contexts, performance-approach goals have also been found related to positive outcomes (Elliot, Cury, Fryer, \& Huguet, 2006; Stoeber et al., 2009).

Achievement goal orientations have been found to vary based on individual differences (Duda \& Nicholls, 1992) and situational factors (Seifritz, Duda, \& Chi, 1992). To examine these variations in goal perspectives within the sport domain, 
studies have investigated achievement goal differences across gender (Abraham, Roberts, \& Pensgaard, 2008; Hanrahan \& Cerin, 2009; Li, Harmer, \& Acock, 1996), sport type (Hanrahan \& Biddle, 2002; Hanrahan \& Cerin, 2009), and level of sport (Fernando-Rio, Estrada, Mendez-Gimenez, Fernandez-Garcia, \& Saavedra, 2014; Lachman, 2014; Yperen \& Renkema, 2008).

The literature demonstrates that achievement goal orientations can be induced by the achievement setting (Papaioannou, Milosis, Kosmidou, \& Tsigilis, 2007). Studies which have compared achievement goal orientations across various types of sport and sport levels present inconsistent findings. In relation to individual versus team sports, Hanrahan and Biddle (2002) found no achievement goal differences across individual and team sports, while Hanrahan and Cerin (2009) found individual sport athletes reporting higher ego orientation than team sport athletes. With respect to the sport level, Hanrahan and Cerin (2009) found no achievement goal differences across recreational and competitive athletes, while Yperen \& Renkema (2008) found higher performance-approach goals among high performing athletes than poor performing athletes, Fernando-Rio and colleagues (2014) found dominant masteryapproach goals among high-level swimmers, and Lachman (2014) found higher ego scores among Division I athletes than Division III athletes.
Recreational sport on college campuses is a critical achievement context as approximately three-fourths of college students use recreation facilities, programs, and services (Forrester, 2014). While most sport participants are motivated by competition, recreational sport is considered a unique population in that competition may be less important than personal improvement (Anderson \& Dixon, 2009). However, with diverse program offerings, the motivation of recreational sport participants may significantly vary based upon program involvement. For example, sport clubs is often perceived as the midpoint between intramural sports and collegiate athletics (Cooney, 1979), suggesting a greater emphasis on competition in sport clubs in comparison to intramural sports. A greater understanding of the motivation of diverse recreation users is instrumental for effective programming that will not only recruit and retain participants but also lead to lifelong recreation participation (Anderson \& Dixon, 2009). Moreover, as research has found achievement goal orientations to be influenced by the achievement setting (Papaioannou et al., 2007), recreation practitioners have the opportunity to enhance optimal achievement goals. Through intentional programming and staff practices, recreation practitioners can influence the achievement goals of their participants in order to enhance positive outcomes. 
Few, if any, studies have examined differences in achievement goals across collegiate recreational sports. Research which has investigated achievement goal variations across individual characteristics and situational factors have predominately used measures reflecting the dichotomous (Task and Ego Orientation in Sport Questionnaire, TEOSQ; Duda, 1989) or 2x2 framework (2x2 Achievement Goals Questionnaire for Sport, AGQ-S; Conroy et al., 2003). The most recent advancement has been the creation of a $3 \times 2$ Achievement Goal Questionnaire for Sport (AGQ-S; Mascret et al., 2014), tested within a Sport Education class in France. The researchers used a convenience sample, limiting the generalizability of the results. Furthermore, the participants were asked to assess achievement goals within the broader context of sport, limiting the precision of the measure. Due to these limitations, it is important to validate an achievement goal measure appropriate for recreational sport participants in the United States prior to testing group differences.

\section{Research Questions}

The $3 \times 2$ achievement goal model is a more rigorous conceptualization of the achievement goal construct. Use of the $3 \times 2$ framework to study achievement goals can offer greater implications for researchers and practitioners. There has yet to be a study which has investigated the achievement goals of recreational sport participants using the $3 \times 2$ conceptual framework. Therefore, a $3 \times 2$ achievement goal scale for recreation sport was developed and tested in the current study, followed by an analysis of achievement goal differences across intramural and club sport groups. Moreover, it was the intention of the researchers to provide a valid tool that can be used by sport researchers and practitioners to gain a greater understanding of the achievement goals of sport participants as well as group differences. In regards to the $3 \times 2$ achievement goal model, the following research questions are made:

R1: Does the $3 \times 2$ achievement goal measurement model demonstrate an acceptable fit for the collegiate recreational sport domain?

R2: Does the $3 \times 2$ achievement goal measurement model demonstrate a better fit than the alternative achievement goal measurement models for the collegiate recreational sport domain?

With respect to achievement goal differences in collegiate recreational sport, the following research question is made: R3: What are the achievement goal differences between individuals with dominant recreation involvement in intramural sports and individuals with dominant recreation involvement in sport clubs?

\section{Method}

Participants and Procedure

The study was conducted at a large, post-secondary institution in the United States. The target population consisted of participants involved in collegiate 
recreational sport programs (i.e., intramural sports and/or sport clubs). A cluster sampling technique was utilized to randomly select 48 intramural sports teams and 32 sport club teams in order to select a representative sample. A total of 727 collegiate recreational sport participants from the selected clusters were invited to participate in the study, of which 722 participated (intramural sport: $n=340$, sport club: $n=382$ ), resulting in a $99.3 \%$ response rate.

As students are able to participate in multiple recreation programs simultaneously, sample 1 (for R1 \& R2) was limited to subjects with dominant participation (greater than $50 \%$ of total recreation involvement) in sport-based recreation programs (i.e., intramural sports and sport clubs) for the purpose of the study $(n=628)$. The threshold of $50 \%$ of total recreation involvement in sport-based recreation programs was used to ensure the participants' perceived achievement goals were reflective of the recreational sport achievement context, as to support the internal validity of the scale for this specific domain. After eliminating ineligible respondents (those without majority participation in sport-based recreation programs) and incomplete cases, sample 1 consisted of 614 subjects. Sample 1 was predominately male $(68.8 \%$ ), with $91.0 \%$ between the ages of 18 to 22 . Of the 614 subjects, $84.7 \%$ self-identified as Caucasian, $4.7 \%$ Asian, $4.5 \%$ "two or more races",
2.4\% Hispanic, 2.3\% African American, and $1.4 \%$ identified other ethnicities.

To compare the measurement model and factor scores across intramural sport and sport club participants, sample 2 (for R3) was limited to subjects with dominant participation (greater than $50 \%$ of total recreation involvement) in one of the two sport-based recreation programs (eliminating participants with equal participation in both programs). Sample 2 consisted of 250 intramural sport participants and 343 sport club participants, for a total of 593 subjects.

All study procedures were approved by the institution's review board prior to data collection. A hard copy questionnaire was administered to the collegiate recreational sport participants at the conclusion of one of their respective intramural sport team competitions (e.g., flag football) or sport club practices or team meetings. To entice participation in the study, an incentive program was administered, for which one subject from each cluster (i.e., intramural sport or sport club team) was randomly selected and given a $\$ 15$ gift card.

\section{Measures}

The instrument consisted of two primary sections focusing on the subjects' recreational sport involvement and achievement goals within the context of collegiate recreational sport. An open-ended quantitative prompt was created to ascertain the subjects' degree of involvement in prominent recreational sport programs (e.g., 
intramural sports, sport clubs) in order to understand their recreation involvement. The subjects were instructed to "record approximately how many hours on a typical week [they] are involved" for each recreation program.

A modified version of the $2 \mathrm{x} 2$ Achievement Goals Questionnaire for Sport (AGQ-S; Conroy et al., 2003) and 3x2 achievement goal model (Elliot et al., 2011) was used to examine a $3 \times 2$ achievement goal framework within the sport context. The $2 \times 2$ framework is comprised of 4 distinct achievement goals measured by 12 items ( 3 items per goal) describing different ways participants approach or avoid competence. The 2x2 AGQ-S demonstrated strong factorial invariance, latent mean stability over a 21-day interval, and external validity established through correlations between achievement goal scores and fear-of-failure scores (Conroy et al., 2003).

The $3 \times 2$ achievement goal model is an extension of the $2 \times 2$ framework in that taskand self-based standards of evaluation are distinguished as unique definitions of mastery competence (Elliot et al., 2011). The $3 \times 2$ framework is composed of six achievement goals from within the context of an academic domain. The model consists of 18 items ( 3 per goal) describing types of goals participants may or may not have for an academic class. Participants are asked to respond on a 7 -point Likert-type scale $(1=$ not true of me $\ldots 7=$ extremely true of me).
Elliot et al. (2011) found the $3 \times 2$ framework to be a good fitting model $\left[x^{2}(120, N=126)=194.25, p<.01, \mathrm{CFI}=\right.$ 0.95$, TLI $=0.94$, RMSEA $=0.070]$. Internal consistency analyses of the achievement goals resulted in relatively high reliability (a $=.77-.93)$. The strongest intercorrelations were found between the approach/avoidance goals within the different standards of evaluation (taskbased, self-based, other-based). The $3 \times 2$ model was tested against 10 alternative models for which the $3 \times 2$ model demonstrated better fit than the alternative models.

The proposed $3 \times 2$ achievement goal scale for recreational sport was developed for the current study from the $3 \times 2$ model, utilizing similar items and scale structure in order to maintain the six achievement goal constructs (see Table 1). The $3 \times 2$ model items were modified to be appropriate for the recreational sport context, in which the sport language (e.g., perform) from the $2 \times 2$ AGQ-S was utilized. The 7-point Likert type scale used within the $3 \times 2$ measure was modified to a 6 -point scale $(1=$ not true of me ... $6=$ very true of me) based upon the argument that inclusion of a no-opinion option inhibits meaningful measurement (Krosnick et al., 2002). However, it should be noted that the no-opinion option may represent genuine dispositions of the respondents and eliminating this option has the potential to inflate the results (Krosnick et al., 2002). Finally, the subjects were asked to respond to the achievement goal items 
within the context of collegiate recreational sport activities.

Once the $3 \times 2$ achievement goal scale for recreational sport was developed, a pilot test was conducted to examine the structure and reliability of the new scale. The instrument was pilot tested with 45 undergraduate students enrolled in the Sport and Leisure Studies program at the large institution studied. The population selected for the pilot study was based upon the expectation that those enrolled in a sport-related major would be likely to participate in collegiate recreational sport. Principal component analysis was conducted to examine the structure of the achievement goal scale, for which four components were extracted based on an eigenvalue greater than one (Stevens, 2009), accounting for $68 \%$ of the variance. The factor loadings were relatively moderate ranging from 0.496 to 0.693 , providing a basis for which items needed modifications. A panel of eight experts including an author of the $2 \times 2$ and $3 \times 2$ achievement goal model, recreational sports professional staff, sport management professors, and experts on quantitative research methodology - reviewed the instrument and provided feedback as to the content validity and soundness of the instrument. A final round of revisions was made to the content of the tool based upon results of the pilot test and feedback from the panel of experts. Random processes were utilized to order the achievement goal items within the scale. The developed achievement goal items and proposed relationships can be found in Table 1 .

\section{Statistical Analysis}

The data were entered into SPSS Statistics 21 software for treatment and analysis. Following data screening, single and multiple imputation data treatment techniques were employed to reduce the amount of missing data. Regression imputation is recommended for a moderate amount of missing values as it is more sophisticated and uses more information when replacing the missing values (Kline, 2011; Schumacker \& Lomax, 2010). However, use of regression imputation may result in biased parameter estimates and underestimated error variance.

Once missing data were treated, the subjects' recreational sport involvement was determined. A total recreation involvement variable was calculated by the sum of involvement in intramural sports and sport clubs. Subjects were then grouped by collegiate recreational sport program (i.e., intramural sports or sport clubs) based upon a criteria of greater than $50 \%$ involvement in one of the two programs. While the combined sample 1 was used for model testing, sample 2, consisting of individual groups, was used for additional CFAs as well as multivariate analysis of variance (MANOVA) testing.

Once the subjects were grouped by recreational sport involvement, the data set was imported to LISREL 9.1 software to produce a correlation matrix of the 
observed variables for CFA and normality statistics (i.e., skewness and kurtosis) of the achievement goal variables. Skewness and kurtosis statistics of the observed variables were calculated, as nonnormal data may affect the parameter estimates, standard errors, and fit indices. Normality was considered to be met if all skewness values fell within the accepted range of +2.00 to 2.00 and kurtosis values fell within the accepted range of +5.00 to -5.00 (Kendall \& Stuart, 1958). Multivariate normality was assessed through relative multivariate kurtosis, for which a value of less than +3.00 was considered indicative of multivariate normality (Siekpe, 2005).

Reliability testing was conducted prior to factor analysis in order to determine if CFA is an appropriate analytic approach. To test the reliability of the proposed achievement goal factors, Cronbach's alpha was calculated for each subscale. The alpha coefficient is one of the most pervasive measures of reliability for studies using psychometric scales in the social sciences (Bonet \& Wright, 2015; Dunn, Baguley, \& Brunsden, 2014). An achievement goal factor was considered reliable with a Cronbach's alpha of 70 or greater (Hair, Anderson, Tatham, \& Black, 1998).

After preliminary testing, the correlation matrix was inputted into LISREL 9.1 software and the model relationships and parameters were specified for further factor analysis. CFAs were conducted to confirm the factor structure and compare the proposed model against the three alternative models. Robust maximum likelihood (MLR) estimation was employed for all analyses, as this method of estimation is recommended for slight to moderate non-normal interval data (Morata-Ramirez \& Holgado-Tello, 2013). When specifying the relationships between the observed and latent variables, all factors were allowed to correlate and the variance of each latent variable was allowed to be 1.0. Upon running the initial model, the largest factor loading for each factor was fixed to 1.0 and the model was run a second time.

To answer R1 and R2, the observed $t$ values for the individual paths in the model were compared to the critical $t$-value for a two-tailed test at the $\alpha<.05$ level of significance. Once statistical significance of the parameter estimates was determined, the fit of the proposed and alternative models were assessed using the following standards of model fit: a nonstatistically significant chi-square $\left(x^{2}\right)$, a root-mean-square error of approximation (RMSEA) less than .100, a goodness-of-fit index (GFI) and comparative fit index (CFI) greater than .95 (.90 is also considered acceptable), and a standardized root-mean-square residual (SRMR) less than .05 (Schumacker \& Lomax, 2010). In order to determine the best fitting model, the parameters, standardized residual matrix, modification indices (MI), expected parameter change (EPC) statistics, and squared multiple correlations $\left(R^{2}\right)$ for each equation of the proposed $3 \times 2$ model were evaluated. The model was considered to be theoretically 
and empirically supported if the majority of fit indices indicated an acceptable model (Schumacker \& Lomax, 2010), and there were no theoretically justifiable modification suggestions. A chi-square difference test was employed to compare the $3 \times 2$ achievement goal model with each alternative model to determine if increasing parsimony significantly deteriorated the model fit (Kline, 2011).

Once the factor structure of the achievement goal measurement model was confirmed, the reliability of each subscale was calculated a second time using Cronbach's alpha. Following reliability testing, the data were reduced into corresponding factor scores. Using SPSS Statistics 21 software, the assumptions for conducting a MANOVA were first examined. In addition to the normality statistics previously outlined, residual plots by groups for each dependent variable were examined, for which unsystematic patterns were indicative of independent observations (Stevens, 2009). Furthermore, homogeneity of variance and covariance for each dependent variable were examined through use of Levene's text and Box's test, with a non-significant result demonstrating homogeneity of variance (Stevens, 2009). Once the assumptions were established, a MANOVA was employed to answer R3. The Wilks $\Delta$ test criteria was adopted to determine whether there was a multivariate significant difference across groups, based on a $\alpha<.05$ level of significance (Stevens, 2009). Upon examination of the main effect, the univariate analyses of variance (ANOVA) were examined using a

Bonferroni correction of $\alpha<.008$, based on six dependent variables (Stevens, 2009).

\section{Results}

\section{Data}

The questionnaire was administered to 727 collegiate recreational sport participants at a large university, of which 628 eligible individuals completed the questionnaire. Data screening revealed that $9 \%$ of cases $(n$ = 57) were incomplete. The Markov chain Monte Carlo (MCMC) multiple imputation method was conducted, resulting in 614 complete cases. Subjects were then grouped by recreational sport program involvement, for which 250 participants were classified within the intramural sport group and 343 within the sport club group.

\section{Normality}

Upon examination of the normality statistics (Table 1), univariate skewness and kurtosis for all observed variables fell within the accepted ranges previously noted (Kendall \& Stuart, 1958). Furthermore, the moderately small value of the relative multivariate kurtosis (1.623) supports the multivariate normality of the data. The normality assumption was concluded to have been met.

\section{Measurement Model}

A correlation matrix of the observed variables was produced (Table 2), and CFA of the proposed $3 \times 2$ model was run to 
answer R1. With 171 unique values in the covariance matrix $\mathrm{S}$ and 51 free parameters, there were 120 degrees of freedom indicating that the model is over-identified and there is room for model modification. All factor loadings were found significant at the $\alpha<.001$ level of significance. The global fit indices of the proposed model $\left[x^{2}(120)=1221.908, \mathrm{p}<.001\right.$; RMSEA: .099; GFI: .857; CFI: .941; SRMR: .036] suggested an acceptable fitting model as three of the five indices met the standards previously noted. Therefore, in response to $\mathrm{R} 1$, the $3 \times 2$ achievement goal measurement model was found to demonstrate an acceptable fit for the collegiate recreational sport domain.

Prior to considering model modifications, the alternative models were tested to answer R2. All achievement goal models were over-identified, for which the $3 \times 2$ and $2 \times 2$ models were found to have an acceptable fit and the trichotomous and dichotomous models were found to be poor fitting (Table 3). Of the four achievement goal models, the $3 \times 2$ framework provided the best fitting model. Chi-square difference tests revealed significant deterioration in model fit for every alternative model. Therefore, in response to $\mathrm{R} 2$, the $3 \times 2$ achievement goal measurement model was found to demonstrate a better fit than the alternative models for the collegiate recreational sport domain.

While the global fit indices for the proposed 3x2 model suggest an acceptable fitting model, with all factor loadings found to be significant, the output indicated possible model modifications. Large standardized residuals were found between Goal 10 and 1 and between Goal 18 and 14. The large residual between Goal 10 and 1 suggest a possible path modification, however the MI does not support this modification. Additionally, the large residual associated with Goal 18 and 14 may suggest a misspecification of correlated measurement error terms. As both variables load onto the same factor, a path modification is not justifiable. Several MI and EPC statistics were provided, of which the modification with the largest anticipated decrease in chi-square (adding a path from Goal 7 to Self-Approach) is not theoretically supported. Therefore, to address the large MI associated with Goal 7, the item was removed for a more parsimonious and better fitting model.

Overall, the modification reduced the standardized residuals and improved the fit of the model, with the majority of fit indices upholding the standards previously noted $\left[x^{2}(104)=961.702, p<.001\right.$; RMSEA: .094; GFI: .880; CFI: .951; SRMR: .034]. The modification suggestions were once again evaluated, for which no changes were found theoretically sound. Thus, the modified model (Figure 1), with three indices indicating a good fit, was found just. The estimated non-centrality parameter (NCP) of the modified model was 857.702 , suggesting a small effect size.

Additional CFA analyses were conducted to determine whether the 
modified model was upheld within different recreational sport contexts. The modified model was found to be an acceptable fit for both the intramural sport group $\left[\chi^{2}(104)=\right.$ 342.67, $p<.001$; RMSEA: .096; GFI: .856; CFI: .936; SRMR: .049] and sport club group $\left[\chi^{2}(104)=310.543, p<.001\right.$; RMSEA: .076; GFI: .906; CFI: .959; SRMR: .036], with the majority of fit indices suggesting good fitting models. Review of the suggested modifications supported no additional changes to the models.

\section{Reliability}

Initial reliability testing of the proposed achievement goal factors demonstrated high reliability ( $\alpha=.846$ - 945). Upon review of item deletion, the results indicated possible issues with Goals 1, 3, and 7 which slightly reduced their respective scale's reliability statistic $(\Delta \alpha=.004-.010)$. Reliability testing was conducted for a second time using the modified achievement goal scales, with results demonstrating high reliability for all factors (i.e., task-approach $\alpha=.875$, task-avoidance $\alpha=.875$, self-approach $\alpha=$ .886, self-avoidance $\alpha=.846$, otherapproach $\alpha=.945$, other-avoidance $\alpha=$ .888). Furthermore, item deletion supported the final factor structure resulting from the CFAs.

\section{Multivariate Analysis of Variance}

Prior to conducting a MANOVA, the assumptions were first examined, with the normality assumption previously established (see Table 1). In regards to the independence assumption, residual plots by groups for each achievement goal variable demonstrated random, not systematic, patterns, which confirmed the assumption. Furthermore, Levene's test revealed nonsignificant values for four of the six achievement goals while Box's test demonstrated significant values for all achievement goals, indicating the homogeneity assumption was partially met. As a MANOVA is relatively robust to a violation of homogeneity with relatively equivalent sample sizes (Stevens, 2009), the data were deemed to have met the necessary assumptions.

Once the assumptions were established, a MANOVA was conducted, upon which the multivariate main effect was found statistically significant $(p<.001)$. When examining the univariate ANOVAs, four of the six achievement goals were found significantly different between intramural and sport club groups (Table 4; Stevens, 2009). The effect sizes were small to medium (partial $n^{2}$ ranging from .02 to .08) and observed power strong (ranging from .96 to 1.00; Stevens, 2009). In response to $\mathrm{R} 3$, of the six achievement goals, the sport club group was found to have significantly greater mastery-based achievement goals. Comparatively, the performance-based achievement goals were not significantly different across groups. 


\section{Discussion}

A 3x2 achievement goal scale for recreational sport was proposed and administered to recreational sport participants at a post-secondary institution. The $3 \times 2$ model was tested against alternative theoretical models to confirm the structure of the measurement model. Of the four theoretical models tested, the $3 \times 2$ model was found to have the best fit. However, each model demonstrated relatively large chi-square values. The significant chi-square statistics are likely a result of the large sample size $(N=614)$ as the chi-square model-fit criterion is sensitive to sample size (Schumacker \& Lomax, 2010). Chi-square is also a function of degrees of freedom (Schumacker \& Lomax, 2010). With 18 observed variables, the number of unique values in the covariance matrix $\mathrm{S}$ was much greater than the number of free parameters for each model. This resulted in large degrees of freedom, consequently affecting the chi-square tests. The large sample size and overidentification of the models are possible reasons for the chi-square index suggesting poor fitting models.

As the $3 \times 2$ achievement goal model was found to have the best fit out of the four theoretical models, modifications to the $3 \times 2$ model were considered to improve the fit of the model. The model was modified to remove Goal 7 from loading onto the taskapproach factor. Of the task-approach items, Goal 7 is the only item with the qualifying phrase "to perform", while the other two task-approach items use the qualifying phrase "to master". This discrepancy may be why Goal 7 was an inadequate measure of task-approach goals. While a modification was made to the initial $3 \times 2$ achievement goal model, the $3 \times 2$ structure of the model was upheld. The modified model was tested within two sport contexts of varying competitive levels (i.e., intramural sport and sport clubs), for which the model was upheld in both contexts. These results support the utility of the model in diverse recreational sport contexts. Overall, the findings of the current study support the most recent theoretical differentiation of achievement goals, which integrate standards of evaluation to define competency (Elliot et al., 2011).

The $2 \times 2$ achievement goal model was also found to have an acceptable fit in comparison to the alternative theoretical models. However, chi-square difference tests revealed significant deterioration in the $3 \times 2$ model when made more parsimonious. This finding supports Elliot et al.'s (2011) supposition that the $3 \times 2$ model provides a better fit than the alternative theoretical models. While parsimony is preferred (Kline, 2011), the $3 \times 2$ framework is considered superior due to conceptual, empirical, and practical advantages. Conceptually, the $3 \times 2$ achievement goal model differentiates task-based and selfbased goals, providing for a more precise measure of mastery goals. Furthermore, as different $2 \times 2$ achievement goal measures have been found to focus on either task- 
based goals, self-based goals, or a combination of both (Mascret et al., 2014), the differentiation found in the $3 \times 2$ framework will support accurate interpretation of the mastery construct. Lastly, more precise information regarding mastery goals will enhance practitioners' ability to intentionally design sport programs, influence the sport environment, and guide participant behaviors. As a whole, the modified $3 \times 2$ achievement goal scale for recreational sport can help practitioners better assess sport participants' achievement goals, which will subsequently inform programmatic decisions.

The multiple theoretical models proposed in achievement goal literature suggest a need to first investigate how to appropriately measure achievement goals within a population before utilizing the construct within a research study.

Practitioners interested in assessing achievement goals among sport participants should also consider their desired conceptualization of perceived competence when selecting an achievement goal tool. Ultimately, the modified $3 \times 2$ achievement goal scale for recreational sport is an appropriate instrument for future research investigating achievement goals within the recreational sport context.

In addition to developing and testing a $3 \times 2$ achievement goal scale for recreational sport, the current study sought to compare distinct achievement goals across collegiate recreational sport programs. The findings demonstrate significantly greater perceived mastery-based goals amongst the sport club group compared to the intramural sport group. Furthermore, non-significant differences in perceived performance-based goals were found between the sport club and intramural sport groups. Overall, these results support Papaioannou and colleagues' (2007) assertion that achievement goal orientations can be influenced by the achievement setting.

When examining the distinct achievement goals, mastery-approach goals were found to be the highest perceived achievement goals for sport club participants, supporting Fernando-Rio and colleagues' (2014) study. However, the significant difference in mastery-based achievement goals across collegiate recreational sport programs is contrary to Hanrahan and Cerin's (2009) finding of no differences across sport levels. Furthermore, the non-significant difference in performance-based goals is not consistent with the findings of Yperen \& Renkema (2008) and Lachman (2014), which demonstrated higher performance-based goals among higher sport levels (i.e., high performing athletes, Division I athletes).

The differences in achievement goals across collegiate recreational sport programs may be indicative of program design differences. In comparison to intramural sports, the sport club program is studentdriven in which the administration and development of the sport is reliant upon student members (Cooney, 1979; Lower et al., 2015). Additionally, participation in 
sport clubs can be characterized by purposeful engagement. Club members pay dues, attend regular practices, and possibly participate in extramural competition, with many teams instructed by a coach or captain (Lower et al., 2013; Mull, Bayless, \&

Jamieson, 2005). The additional responsibility of club operations, meetings, and practice is likely to enhance task- and self-based achievement goals.

Comparatively, intramural sport involvement is often limited due to program restrictions (e.g., registration limitations, sport schedules, number of sports administered simultaneously; Lower et al., 2015). Intramural sport teams are unlikely to have a coach and rarely meet outside of competitions for practice. The primary emphasis of the intramural sport program is competition, for which participants can choose among a variety of leagues associated with different skill levels (Lower et al., 2015). These program design differences may explain the greater masterybased achievement goals reported by sport club participants in comparison to intramural sport participants.

When comparing sport level, sport clubs have been characterized as the median between intramural sport and collegiate athletics (Cooney, 1979). Furthermore, the intramural sport program typically consists of multiple sport leagues corresponding with varying skill levels and interests (Lower et al., 2015). Intramural sport participants might be characterized as recreational or competitive. While the sport club program is often considered a higher sport level, the current study found non-significant differences in perceived performance-based achievement goals across sport club and intramural sport groups. This finding may be due to intramural sport's exclusive focus on competition, rather than the congruent pursuit of development and competition found in sport clubs.

Overall, the current study demonstrates differential achievement goals across collegiate recreational sport programs. Research has predominantly supported mastery- and approach-based goals as antecedents of positive outcomes in sport (Kavussanu et al., 2011; Lower et al., 2014; Spray et al., 2006). Therefore, recreational sport practitioners should examine the structure and design of their programs to determine how the achievement settings might influence participants' achievement goals. Through intentional programming, practitioners can induce distinct goal orientations, thus indirectly enhancing positive outcomes. Programming that incorporates goal setting, instruction, and skill modifications may enhance mastery goals. Furthermore, the use of positive framing, constructive feedback, and management of maladaptive behaviors (e.g., avoidance) by sport coaches and captains may enhance approach goals (Lower et al., 2014).

\section{Limitations and Recommendations}

The $3 \times 2$ achievement goal scale for recreational sport was developed from 
existing measures and pilot tested once prior to its administration in the current study. The first pilot test extracted four factors from the scale as previously noted. Subsequent pilot tests were not conducted to examine the structure of the modified tool, limiting the reliability of the proposed scale. The proposed $3 \times 2$ achievement goal model was modified to achieve a better fit. The removal of Goal 7 altered the intended structure of the achievement goal scale (i.e., three items per factor). Future research may want to consider revising Goal 7 to more effectively measure the task-approach goal and therefore maintain the three items per factor scale structure.

Based upon the results of the current study, the six-factor structure was supported over the alternative theoretical models. The more complex conceptualization of achievement goals can provide a greater depth of understanding regarding a sport participant's motivation and perception of competence. Additionally, the greater precision can offer more effective predictors of achievement related outcomes. The $3 \times 2$ achievement goal scale for recreational sport should continue to be tested, within various sport contexts, to confirm or disconfirm the $3 \times 2$ model structure proposed. Another consideration is the measurement of achievement goals at one point in time. As achievement goals have been found to vary based on environmental cues (Seifritz et al., 1992), they are likely to change over time and not remain constant. Future studies should seek to account for the possible change in achievement goal orientations across achievement goal settings and across time.

The current study had an exclusive focus on recreational sport participants at a single university, limiting the generalizability of the study's results. Future research should consider inclusion of multiple institutions or comparison of different sport domains (e.g., community, recreational, collegiate) for a particular demographic. Based upon the nature of recreation, many collegiate recreational sport participants engage in multiple diverse programs simultaneously. Subsequent studies could assess how these dynamic recreation users' achievement goals differ based on the achievement context. This line of inquiry will provide a greater understanding of the influence of program design on achievement goal orientations. In the end, the $3 \times 2$ achievement goal model was upheld for the sport population studied, supporting the most recent theoretical model (Elliot et al., 2011). Future research may consider including antecedents or consequences of achievement goals when utilizing the modified $3 \times 2$ achievement goal scale for recreational sport. Expanding our understanding of the motivation of sport participants can provide greater implications for practitioners. 


\section{Acknowledgements}

This work was supported by funds

received from the North American Society

for Sport Management (NASSM) Research

Grant Program. 


\section{References}

Abraham, F. E., Roberts, G. C., \& Pensgaard, A. M. (2008).

Achievement goals and gender effects on multidimensional anxiety in national elite sport. Psychology of Sport \& Exercise, 9(4), 449-464. doi:10.1016/j.psychsport.2007.06.005

Adie, J. W., Duda, J. L., \& Ntoumanis, N. (2010). Achievement goals, competition appraisals, and the welland ill-being of elite youth soccer players over two competitive seasons. Journal of Sport \& Exercise Psychology, 32(4), 555-579.

Adie, J. W., \& Jowett, S. (2010). Metaperceptions of the coach-athlete relationship, achievement goals, and intrinsic motivation among sport participants. Journal of Applied Social Psychology, 40(11), 2750-2773.

Anderson, D. M., \& Dixon, A. W. (2009).

Winning isn't everything: Goal orientation and gender differences in university leisure-skills classes. Recreational Sports Journal, 33, 54-64.

Blumenthal, K. J. (2009). Collegiate recreational sports: Pivotal players in student success. Planning for Higher Education, 37(2), 52-62.

Bonett, D. G., \& Wright, T. A. (2015). Cronbach's alpha reliability: Interval estimation, hypothesis testing, and simple size planning. Journal of Organizational Behavior, 36, 3-15.

Cervelló, E. M., Excartí, A., \& Guzmán J. F. (2007). Youth sport dropout from the achievement goal theory. Psicothema, 19, 65-71.

Conroy, D. E., Elliot, A. J., \& Hofer, S. M. (2003). A 2 x 2 achievement goals questionnaire for sport: Evidence for factorial invariance, temporal stability, and external validity. Journal of Sport and Exercise Psychology, 25(4), 456-476.

Cooney, L. (1979). Sport clubs: Their place within the total intramuralrecreational sports program. Journal of Physical Education and Recreation, 50(3), 40-41.

Cury, F., Elliot, A. J., Fonseca, D. D., \& Moller, A. C. (2006). The socialcognitive model of achievement motivation and the $2 \times 2$ achievement goal framework. Journal of Personality and Social Psychology, 90(4), 666-679.

de Bruin, A. P., Bakker, F. C., \& Oudejans, R. R. D. (2009). Achievement goal theory and disordered eating: Relationships of disordered eating with goal orientations and motivational climate in female gymnasts and dancers. Psychology of Sport \& Exercise, 10(1), 72-79. doi:10.1016/j.psychsport.2008.07.002

Duda, J. L. (1989). Relationship between task and ego orientation and the perceived purpose of sport among high school athletes. Journal of Sport and Exercise Psychology, 11, 318-335.

Duda, J. L., \& Nicholls, J. G. (1992). Dimensions of achievement motivation in schoolwork and sport. Journal of Educational Psychology, 84, 290-299.

Dunn, T. J., Baguley, T., \& Brunsden, V. (2014). From alpha to omega: A practical solution to the pervasive problem of internal consistency estimation. British Journal of Psychology, 105, 399-412. 
Dweck, C. S., \& Leggett, E. L. (1988). A social-cognitive approach to personality and motivation. Psychological Review, 95, 256-273.

Elliot, A. J. (1999). Approach and avoidance motivation and achievement goals. Educational Psychologist, 34(3), 169-189. doi:10/1207/s15326985ep3403_3

Elliot, A. J. (2005). A conceptual history of the achievement goal construct. In A. J. Elliot \& C. S. Dweck (Eds.), Handbook of competence and motivation (pp. 52-72). New York, NY: The Guilford Press.

Elliot, A. J., \& Covington, M. V. (2001). Approach and avoidance motivation. Educational Psychology Review, 13(2), 7392.

Elliot, A. J., Cury, F., Fryer, J. W., \& Huguet, P. (2006). Achievement goals, self-handicapping, and performance attainment: A mediational analysis. Journal of Sport \& Exercise Psychology, 28, 344-361.

Elliot, A. J., \& Harackiewicz, J. M. (1996). Approach and avoidance achievement goals and intrinsic motivation: A meditational analysis. Journal of Personality and Social Psychology, 70(3), 461-475.

Elliot, A. J., \& McGregor, H. A. (2001). A 2x2 achievement goal framework. Journal of Personality and Social Psychology, 80(3), 501-519. doi:10.1037//0022-3514.80.3.501

Elliot, A. J., Murayama, K., \& Pekrun, R. (2011). A $3 \times 2$ achievement goal model. Journal of Educational Psychology, 103(3), 632-648.

Fernando-Rio, J., Estrada, J. A. C., MendezGimenez, A., Fernandez-Garcia, B.,
\& Saavedra, P. (2014). $2 \times 2$ dominant achievement goal profiles in high level swimmers. European Journal of Sport Science, 14(3), 265-272. doi:10.1080/17461391.2013.819383

Forrester, S. (2014). The benefits of campus recreation. Corvallis, OR: NIRSA.

Gilson, T. A., Chow, G. M., \& Ewin, M. E. (2008). Using goal orientations to understand motivation in strength training. Journal of Strength and Conditioning Research, 22(4), 1169-1175. doi:10.1519/JSC.0b013e318173c566 Hair, J. F., Anderson, R. E., Tatham, R. L., \& Black, W. C. (1998). Multivariate data analysis with readings. Englewood, NJ: Prentice Hall.

Hanrahan, S. J., \& Biddle, S. J. H. (2002). Measurement of achievement orientations: Psychometric measures, gender, and sport differences. European Journal of Sport Science, 2(5), 1-12. doi:10.1080/17461390200072502 Hanrahan, S. J., \& Cerin, E. (2009). Gender, level of participation, and type of sport: Differences in achievement goal orientation and attributional style. Journal of Science and Medicine in Sport, 12, 508-512. doi:10.1016/j.jsams.2008.01.005

Kavussanu, M., \& Morris, R. L. (2009). The role of approach-avoidance versus task and ego goals in enjoyment and cognitive anxiety in youth sport. International Journal of Sport and Exercise Psychology, 7(2), 185-202.

doi:10.1080/1612197X.2009.9671899

Kavussanu, M., White, S. A., Jowett, S., \& England, S. (2011). Elite and nonelite male footballers differ in goal 
orientation and perceptions of parental climate. International Journal of Sport \& Exercise Psychology, 9(3), 284290. doi:10.1080/1612197X.2011.614854

Kendall, M. G., \& Stuart, A. (1958). The advanced theory of statistics. New York, NY: Hafner.

Krosnick, J. A., Holbrook, A. L., Berent, M. K., Carson, R. T., Hanemann, W. M., Kopp, R. J., ... Conaway, M. (2002). The impact of "no opinion" response options on data quality: Non-attitude reduction or an invitation to satisfice? Public Opinion Quarterly, 66(3), 371403. doi:10.1086/341394

Lachman, M. (2014). Antecedents of athletes' achievement goal orientations (Masters thesis). Retrieved from Digital Commons. (1134)

LeUnes, A. (2008). Sport psychology (4th ed.). New York, NY: Psychology Press.

Li, F., Harmer, P., \& Acock, A. (1996). The task and ego orientation in sport questionnaire: Construct equivalence and mean differences across gender. Research Quarterly for Exercise and Sport, 68, 228-238.

Lower, L. M., Turner, B. A., \& Petersen, J. C. (2015). Does greater opportunity for recreational sport involvement translate to greater degree of involvement? International Journal of Sport Management, 16(1), 62-76.

Lower, L. M., Turner, B. A., \& Petersen, J. C. (2014). Achievement goal orientation as a predictor of sport involvement and perceived benefits of sport involvement: Examination of a mixture model. Journal of SPORT, 3(1), 63-101.
Lower, L. M., Turner, B. A., \& Petersen, J. C. (2013). A comparative analysis of perceived benefits of participation between recreational sport programs. Recreational Sports Journal, 37, 66-83.

Mascret, N., Elliot, A. J., \& Cury, F. (2014). Extending the $3 \times 2$ achievement goal model to the sport domain: The $3 \times 2$ Achievement Goal Questionnaire for Sport. Psychology of Sport and Exercise, 17, 7-14.

Morata-Ramirez, M. A., \& Holgado-Tello, F. P. (2013). Construct validity of likert scales through confirmatory factor analysis: A simulation study comparing different methods of estimation based on pearson and polychoric correlations. International Journal of Social Science Studies, 1(1), 5461.

Mull, R. F., Bayless, K. G., \& Jamieson, L. M. (2005). Recreational sport management (4th ed.). Champaign, IL: Human Kinetics.

Nicholls, J. G. (1984). Achievement motivation: Conceptions of ability, subjective experience, task choice, and performance. Psychological Review, 91(3), 328-346.

Nicholls, J. G. (1989). The competitive ethos and democratic education. Cambridge, MA: Harvard University Press.

Nicholls, J. G., \& Dweck, C. S. (1979). A definition of achievement motivation. Unpublished manuscript, University of Illinois, Champaign, IL.

Nien, C., \& Duda, J. L. (2008). Antecedents and consequences of approach and avoidance achievement goals: A test of gender invariance. Psychology of Sport and Exercise, 9, 352-372. 
Papaioannou, A. G., Milosis, D., Kosmidou, E., \& Tsigilis, N. (2007). Motivational climate and achievement goals at the situational level of generality. Journal of Applied Sport Psychology, 19, 38-66. doi:10.1080/10413200601113778

Riou, F., Bioché, J., Doron, J., Romain, A. J., Ninot, G., Gernigon, C., ... D'Arripe-Longueville, F. (2012). Development and validation of the French Achievement Goal Questionnaire for Sport and Exercise (FAGQSE). European Journal of Psychological Assessment, 28, 313-320.

Roberts, G. C., Treasure, D. C., \& Conroy, D. E. (2007). Understanding the dynamics of motivation in sport and physical activity: An achievement goal interpretation. In G.

Tenenbaum, \& R. Eklund (Eds.), Handbook of sport psychology (3rd ed.; pp. 3-30). Hoboken, NJ: John Wiley $\&$ Sons, Inc.

Schumacker, R. E. \& Lomax, R. G. (2010). $A$ beginner's guide to structural equation modeling (3rd ed.). New York, NY: Routledge.

Seifritz, J., Duda, J. L., \& Chi, L. (1992). The relationship of perceived motivational climate to achievementrelated affect and cognitions in basketball. Journal of Sport \& Exercise Psychology, 14, 375-391.

Siekpe, J. S. (2005). An examination of the multidimensionality of flow construct in a computer-mediated environments. Journal of Electronic Commerce Research, 6(1), 31-43.

Spray, C. M., Wang, C. K. J., Biddle, S. J. H., \& Chatzisarantis, N. L. D. (2006). Understanding motivation in sport:
An experimental test of achievement goal and self determination theories. European Journal of Sport Science, 6(1), 43-51. doi:10.1080/17461390500422879

Stevens, J.P. (2009). Applied multivariate statistics for the social sciences (5th ed.). New York, NY: Routledge.

Stoeber, J., Uphill, M. A., \& Hotham, S. (2009). Predicting race performance in triathlon: The role of perfectionism, achievement goals, and personal goal setting. Journal of Sport \& Exercise Psychology, 31(2), 211 245.

Wang, C. K. J., Biddle, S. J. H., \& Elliot, A. J. (2007). The $2 \times 2$ achievement goals framework in a physical education context. Psychology of Sport and Exercise, 8, 147-168.

Yperen, N. W. V. (2006). A novel approach to assessing achievement goals in the context of the $2 \times 2$ framework: Identifying distinct profiles of individuals with different dominant achievement goals. Personality and Social Psychology Bulletin, 32(11), 14321445.

Yperen, N. W. V., \& Renkema, L. J. (2008). Performing great and the purpose of performing better than others: On the recursive of the achievement goal adoption process. European Journal of Social Psychology, 38, 260-271. doi:10.1002/ejsp.425 
Table 1

\section{Tables}

Descriptive Statistics of Achievement Goal Observed V ariables $(N=614)$

\begin{tabular}{|c|c|c|c|c|}
\hline Observed Variables & $M$ & $S D$ & Skewness & Kurtosis \\
\hline \multicolumn{5}{|c|}{ Task-Approach } \\
\hline Goal 7: "To perform the task elements well." & 4.915 & 1.118 & -0.920 & 0.400 \\
\hline $\begin{array}{l}\text { Goal 18: "To master my performance of the } \\
\text { task." }\end{array}$ & 4.819 & 1.239 & -0.976 & 0.456 \\
\hline $\begin{array}{l}\text { Goal 14: "To master the aspects of my } \\
\text { performance." }\end{array}$ & 4.799 & 1.237 & -0.918 & 0.244 \\
\hline
\end{tabular}

\section{Task-Avoidance}

Goal 16: "To avoid failing to master the task.” $4.362 \quad 1.471 \quad-0.570 \quad-0.630$

Goal 4: "To avoid performing the task elements $\quad \begin{array}{llll}4.533 & 1.400 & -0.806 & -0.109\end{array}$ poorly."

Goal 15: "To avoid performing the task $\quad \begin{array}{lllll}4.554 & 1.346 & -0.753 & -0.189\end{array}$ poorly."

\section{Self-Approach}

Goal 11: "To perform better than I have in the $\begin{array}{llll}4.955 & 1.090 & -0.842 & 0.023\end{array}$ past."

Goal 1: "To perform well relative to how well I $\quad \begin{array}{lllll}4.986 & 1.148 & -1.023 & 0.384\end{array}$ have performed in the past."

Goal 6: "To perform better than I typically do." $4.920 \quad 1.101 \quad-0.841 \quad 0.045$

\section{Self-Avoidance}

Goal 12: "To avoid performing worse than I $\quad \begin{array}{llll}4.615 & 1.334 & -0.771 & -0.190\end{array}$ typically do."

Goal 8: "To avoid performing poorly compared $4.710 \quad 1.302 \quad-0.921 \quad 0.146$ to my typical level of performance."

Goal 2: "To avoid performing worse than I $\quad \begin{array}{llll}4.644 & 1.475 & -0.941 & -0.060\end{array}$ have in the past." 
Table 1 continued

\section{Other-Approach}

Goal 10: "To perform better than others." $\quad 4.484 \quad 1.435 \quad-0.751 \quad-0.308$

Goal 9: "To do well compared to others." $\quad 4.552 \quad 1.360 \quad-0.768 \quad-0.179$

Goal 5: "To do better than most other $\quad \begin{array}{lllll}4.507 & 1.399 & -0.711 & -0.301\end{array}$ performers."

\section{Other-Avoidance}

Goal 13: "To avoid performing worse than

$\begin{array}{llll}4.372 & 1.513 & -0.641 \quad-0.572\end{array}$

everyone else."

Goal 3: "To avoid performing poorly in $\quad \begin{array}{lllll}4.396 & 1.548 & -0.721 & -0.523\end{array}$

comparison to others."

Goal 17: "To avoid being one of the worst $\quad \begin{array}{lllll}4.481 & 1.527 & -0.757 & -0.485\end{array}$

performers in the group."

Note: Goal 7 was removed from the final $3 \times 2$ achievement goal scale for recreational sport. 


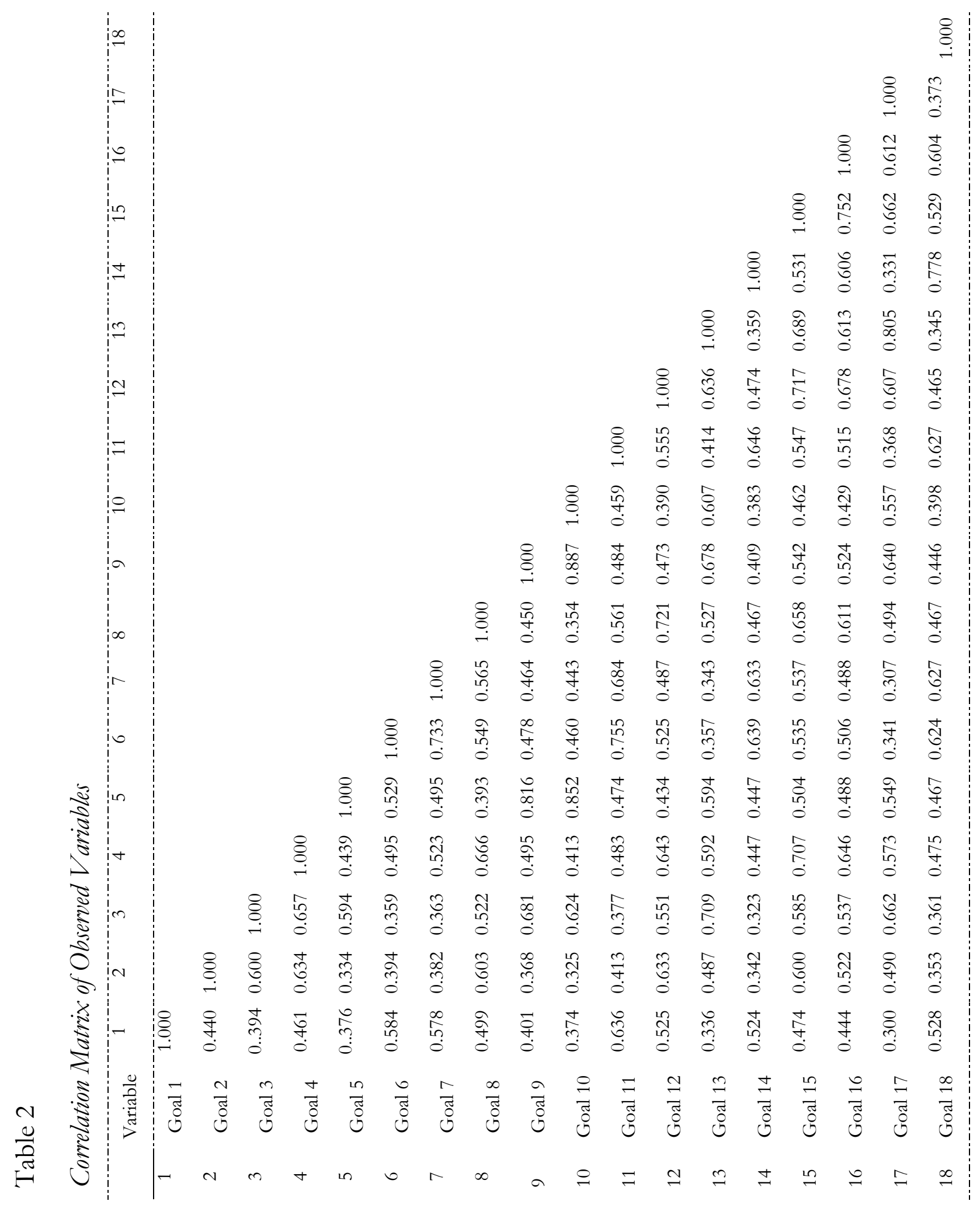


Table 3

Structural Equation Modeling Goodness of Fit Statistics for Theoretical Measurement Models

\begin{tabular}{lccccccc}
\hline Models & $x^{2}$ & $d f$ & $p$ & RMSEA & GFI & CFI & SRMR \\
\hline $3 \times 2$ model & 1221.91 & 120 & $<.001$ & 0.099 & 0.857 & 0.941 & 0.036 \\
$2 \times 2$ model & 1561.11 & 129 & $<.001$ & 0.109 & 0.818 & 0.923 & 0.039 \\
Trichotomous model & 3152.86 & 132 & $<.001$ & 0.157 & 0.639 & 0.837 & 0.071 \\
Dichotomous model & 3871.90 & 134 & $<.001$ & 0.173 & 0.576 & 0.799 & 0.080
\end{tabular}

Note. RMSEA: root-mean-square error of approximation; GFI: goodness-of-fit index; CFI:

comparative fit index; SRMR: standardized root-mean-square residual.

Table 4

MANOVA Univariate Effects (N = 593)

\begin{tabular}{lcccc} 
Achievement Goals & $\begin{array}{c}\text { IM Sports } \\
M(S D)\end{array}$ & $\begin{array}{c}\text { Sport Clubs } \\
M(S D)\end{array}$ & $F(1,591)$ & $p$ \\
\hline Task-Approach (Items: 14, 18) & $4.43(1.26)$ & $5.09(0.99)$ & 50.44 & $<.001$ \\
Task-Avoidance (Items: 4, 15, 16) & $4.26(1.27)$ & $4.64(1.22)$ & 12.90 & $<.001$ \\
Self-Approach (Items: 7, 6, 1, 11) & $4.72(1.04)$ & $5.14(0.86)$ & 28.89 & $<.001$ \\
Self-Avoidance (Items: 12, 8, 2) & $4.46(1.22)$ & $4.82(1.14)$ & 14.02 & $<.001$ \\
Other-Approach (Items: 9, 10, 5) & $4.45(1.25)$ & $4.56(1.36)$ & 0.97 & 0.326 \\
Other-Avoidance (Items: 3, 13, 17) & $4.33(1.33)$ & $4.49(1.40)$ & 1.80 & 0.180
\end{tabular}

Note. Bonferroni correction of $\alpha<.008$ applied.

Journal of Amateur Sport Volume Two, Issue Two

Lower and Turner, 2016 


\section{Figures}

Figure 1

Path diagram of modified $3 \times 2$ achievement goal model with standardized solutions

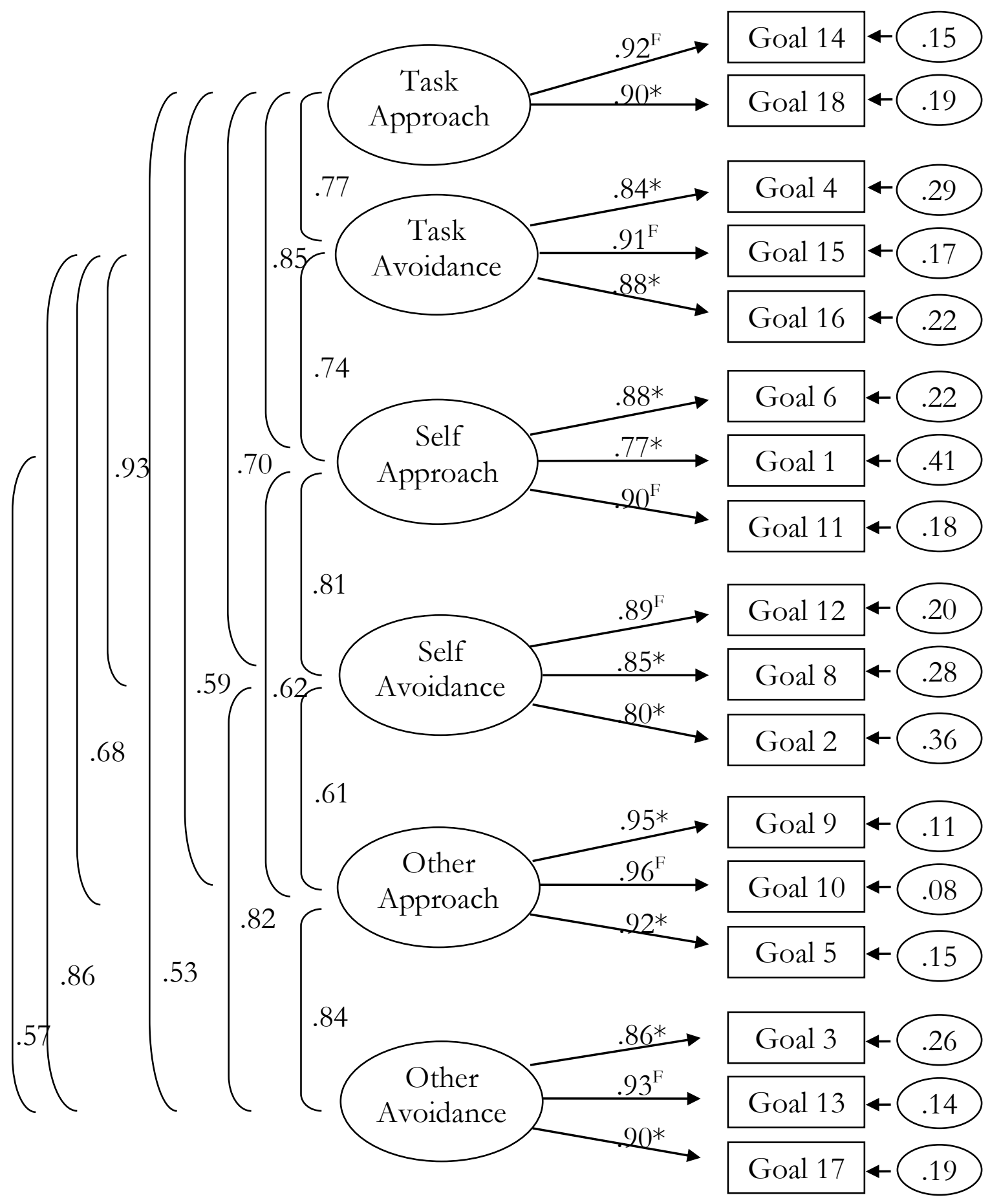

Note. The fixed factors are denoted by ' $\mathrm{F}^{\prime} . * p<.001$.

Journal of Amateur Sport Volume Two, Issue Two

Lower and Turner, 2016 